\title{
True positive fraction
}

Margaret Pepe

\section{Source}

Margaret Sullivan Pepe. (2003). The Statistical Evaluation of Medical Tests for

Classification and Prediction Oxford University Press

The fraction (percentage) of subjects with a given disease correctly identified as such.

Also known as true positive rate or sensitivity. 